\title{
Sprawl angle in simplified models of vertical climbing: Implications for robots and roaches
}

\author{
Goran A. Lynch, ${ }^{\mathrm{a}, *}$, Lawrence Rome ${ }^{\mathrm{b}}$ and Daniel E. Koditschek ${ }^{\mathrm{a}}$ \\ ${ }^{a}$ Department of Electrical and Systems Engineering, University of Pennsylvania, Philadelphia, PA, USA \\ ${ }^{\mathrm{b}}$ Department of Biology, University of Pennsylvania, Philadelphia, PA, USA
}

\begin{abstract}
Empirical data taken from fast climbing sprawled posture animals reveals the presence of strong lateral forces with significant pendulous swaying of the mass center trajectory in a manner captured by a recently proposed dynamical template $[1,2]$. In this simulation study we explore the potential benefits of pendulous dynamical climbing in animals and in robots by examining the stability and power advantages of variously more and less sprawled limb morphologies when driven by conventional motors in contrast with animal-like muscles. For open loop models of gait generation inspired by the neural-deprived regimes of high stride-frequency animal climbing, our results corroborate earlier hypotheses that sprawled posture may be required for stability. For quadratic-in-velocity power output actuation models typical of commercially available electromechanical actuators, our results suggest the new hypothesis that sprawled posture may confer significant energetic advantage. In notable contrast, muscle-powered climbers do not experience an energetic benefit from sprawled posture due to their sufficiently distinct actuator characteristics and operating regimes. These results suggest that the potentially significant benefits of sprawled posture climbing may be distinctly different depending upon the details of the climbers sensorimotor endowment. They offer a cautionary instance against mere copying of biology by engineers or rote study of physical models by biologists through this reminder of how even simple questions addressed by simple models can yield nuanced answers that only begin to hint at the complexity of biological designs and behaviors.
\end{abstract}

Keywords: Bioinspired robotics, climbing robots, robot actuator models, robotic models of animal locomotion

\section{Introduction}

\subsection{Biological inspiration}

For some time, biologists have developed and legged robots have been inspired by simplified, abstracted models of animal locomotion. As described in [1], a template is one such abstraction, comprised of a low dimensional mechanical model whose dynamics replicate the ground reaction force and center of mass

*Corresponding author: Goran A. Lynch, Electrical and Systems Engineering, University of Pennsylvania, Philadelphia, PA 19104, USA. E-mail: goran@seas.upenn.edu. motion patterns exhibited by a designated group of animal species while capable of being dynamically anchored [1] in their detailed morphology in a manner discussed at length in ref. [3]. Perhaps the most widely used template for animal running is the "SpringLoaded Inverted Pendulum," a model which describes the saggital plane motion of all animals whose level running ground reaction forces have been measured [4]. The "Lateral Leg Spring" model [5] was inspired by biological hypotheses about the passive mechanical stabilizing effects of sprawled posture [6] in the horizontal plane. In this paper we are principally concerned with a template for animals running on vertical surfaces [2] which we will discuss in some detail below. 
Animals have many degrees of freedom and impressive muscle power density. ${ }^{1}$ While these aspects of animal morphology are hard or impossible to synthetically replicate or control, templates may be used to guide the design and control of robots [9, 10]. Instead of mimicking the form of animals, designers can target and achieve the motion patterns of the appropriate biological template [11]. When effectively anchored [10], the resulting robotic behavior recovers the targeted features of animal motion without necessitating the animal's morphological complexity. This research methodology provokes the investigation of a central issue: what are the benefits, to animal or to machine, of operating in accordance with a given template?

\subsection{Bioinspired dynamic climbing}

A number of robotics groups have, in recent times, endeavored to build dynamic climbing platforms [12, 13]. Complementing this engineering work, Goldman and Full et al. published several papers investigating the dynamic vertical locomotion of geckos and cockroaches, culminating with the proposal of a template which captures the center of mass motion and ground reaction force patterns of the animals studied [2]. DynoClimber is a dynamic vertical climbing robot which anchored this 2-D biological template [14].

Perhaps the most notable discovery in this biological research [2] was the presence of large lateral forces in both gecko and cockroach climbers. Both climbing geckos and cockroaches pulls sideways with up to $50 \%$ of the force they apply downward. In consequence, both animals' mass center trajectories exhibit oscillatory lateral motions while they ascend vertical surfaces. The Full-Goldman climbing template

\footnotetext{
${ }^{1}$ [7], for instance, presents data that frog muscle has a powe density of $371 \mathrm{~W} / \mathrm{kg}$. In comparison, the motors used on the robot DynoClimber [8] have a peak power density of roughly $500 \mathrm{~W} / \mathrm{kg}$ when operated at $30 \mathrm{~V}$. However, this comparison is compromised by a number of compounding factors: neither muscle nor motor power densities consider a) the mass of the requisite power supply (circuitry and batteries in the case of motors, a circulatory system and host of organs in the case of muscles), b) the mass of a force transmission structure (gearbox and transmission in engineering, connective tissue and skeleton in biology), c) an engineering notion of "maximal excitation" (motors can operate at a wide range of voltages), d) the time period over which the given power density can be produced (to guard against motor damage, for instance, DynoClimber actually climbs with its motors operating at a power density of less than $150 \mathrm{~W} / \mathrm{kg}$ ). Thus it is with a measure of caution that we present any power density comparison, as a principled study of this topic would require a host of additional considerations.
}

addresses this phenomenon by introducing a "sprawl angle" (corresponding to the angle from vertical of the characteristic ground reaction force vectors) upon the hanging spring-loaded pendulum used to abstract the mechanics in the proposed template. In [2], a sprawl angle of roughly $10^{\circ}$ caused the template to match both animals' climbing characteristics admirably.

The production of large lateral forces during vertical climbing is counterintuitive: applying force in any non-vertical direction seems to be a waste of energy. The hypothesis proposed and defended in [2] was one of stability. By employing a nonzero sprawl angle, the template was shown in simulation to recover more quickly from lateral perturbations to its steadystate climbing. In this paper, we address that stability hypothesis and introduce a second, complementary hypothesis for the generation of lateral forces while climbing.

Simulations conducted specific to the design of DynoClimber indicate that increasing sprawl angle may improve vertical climbing speed [15]. We further probe the effects of sprawl angle on power production in a generalized model of climbing which allows us to analyze the effects of both synthetic and biological actuators. In particular, we investigate the conditions under which its morphology allows a climber to operate at or near its peak power speed. We propose the hypothesis that, by employing a sprawl angle, climbers are able ascend vertically at higher rates of speed than they are able to achieve by moving directly vertical. In essence, we investigate the extent to which sprawl acts as a gear ratio.

In order to address the two hypotheses associated with lateral force generation in climbing, we examine two pairs of modeling choices: "sensed" or "blind" leg placement paired with muscle- or motor- actuation.

\subsection{Organization}

In Section 2, we introduce the two leg placement schemes as well as their underlying physical intuition. In Section 3 we develop the two actuator models and justify relevant parameter choices. Section 4 presents the simulation results and 5 summarizes the insight both biological and robotic - that the models provide.

\section{Reduced order templates}

The Full-Goldman template [2] effectively models the steady-state climbing of geckos and cockroaches. Just as has been done for SLIP [16, 17], we reduce this 
model further to isolate the interplay between actuator dynamics and sprawl angle. Thus, we eliminate the rigid body components of the Full-Goldman template and replace them with a single point mass and massless legs. We also eliminate the template's "wrist spring." This feature is critical in the Full-Goldman template, as it smoothes attachment force. However, it also adds complexity to the model and distracts from our focus on the the power-generating actuator. Finally, whereas the Full-Goldman template's actuator simply moves as a prescribed trajectory in time, our models are driven by state (not time) dependent muscle or motor models. Maximizing power output requires actuators to operate near or at their power limits (as in [18]), coupling actuator and system dynamics, hence our emphasis on actuation. This section introduces two similar reduced order templates which exhibit notably different behaviors. The detailed equations of motion are presented in the appendix.

\subsection{Body-switched reduced-order template}

The body-switched reduced-order template is a kinematically simple hybrid model on a vertical plane. It consists of a single point mass body and two massless legs. Each identical leg has a segment of fixed length $l_{b}$, and an actuated segment which can vary in length from a maximum (at the beginning of the stride) of $l_{s}$ to a length of 0 when the leg is fully retracted. The two legs are rigidly separated by an angle of $2 \cdot \psi$, where $\psi$ defines the sprawl angle. This scheme can be thought of as sensorless, as leg placement is constrained-presumably mechanically—by a fixed angle. The body-switched climb is thus named: the relationship of its legs is constrained in "body" coordinates irrespective of the external world.

The climber is attached to the wall by, and free to rotate around, exactly one foot at any point in time. The attached leg's actuator shortens throughout the stride from a length of $l_{s}$ to a length of 0 . A given actuator's collapse to a length of 0 signals the end of a stride and immediately detaches that leg from the wall, while the other leg simultaneously attaches in a fully extended state (ie. with actuator length of $l_{s}$ ). We do not address the extension of each leg in its respective flight phase, as the legs are massless and therefore do not affect body dynamics when unattached to the wall. The model assumes that there is a mechanism in place which ensures each leg is fully extended at the start of its stance phase. A depiction of the climber in mid-stride is included in Fig. 1.

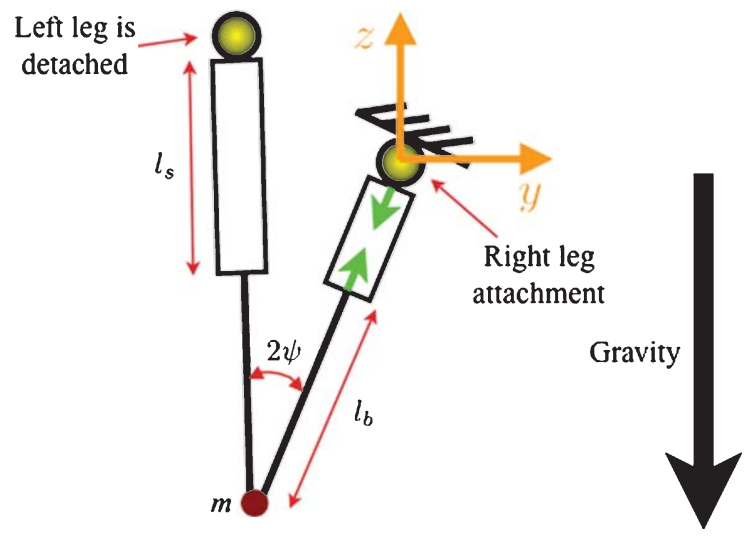

Fig. 1. Mid-stride for the body-switched model. The right foot is attached and serves as a frictionless pivot while the right leg is shortening, while the left foot is detached and its actuator is extended to its maximum length awaiting the next stride.

This model and the Full-Goldman template both employ a rigid sprawl angle; as such, the bodyswitched climber is appropriate for addressing the stability hypothesis. As we demonstrate in Section 4, however, this model is limited in its ability to address the energetic aspects of sprawl. Biologically speaking, the body-oriented switching scheme corresponds to an animal which climbs with limited neural control and simply places each leg (or pair or triplet of legs) and pulls at a certain effective angle.

\subsection{World-switched reduced-order template}

The world-switched variant of our climber is indistinguishable from the body-switched model within a stride. Just as in the body-switched model, when a leg completes its stride and detaches, the other leg instantaneously and simultaneously attaches to the wall. The distinction between models lies in the placement of the "new" stance leg at the end of one stride and the beginning of another. Whereas the body-switched model enforces a constant angular relationship between the two legs, the world-switched climber places its new stance leg at an angle of $\psi$ from vertical at the beginning of each stride, irrespective of the former stance leg's position, as depicted in Fig. 2. Again, the angular position of the inactive leg does not affect system dynamics, since the legs are massless.

The sprawl angle in this model has a slightly different manifestation than sprawl angle in the bodyswitched model, but qualitatively both sprawl angles have a similar effect: the presence of sprawl induces 


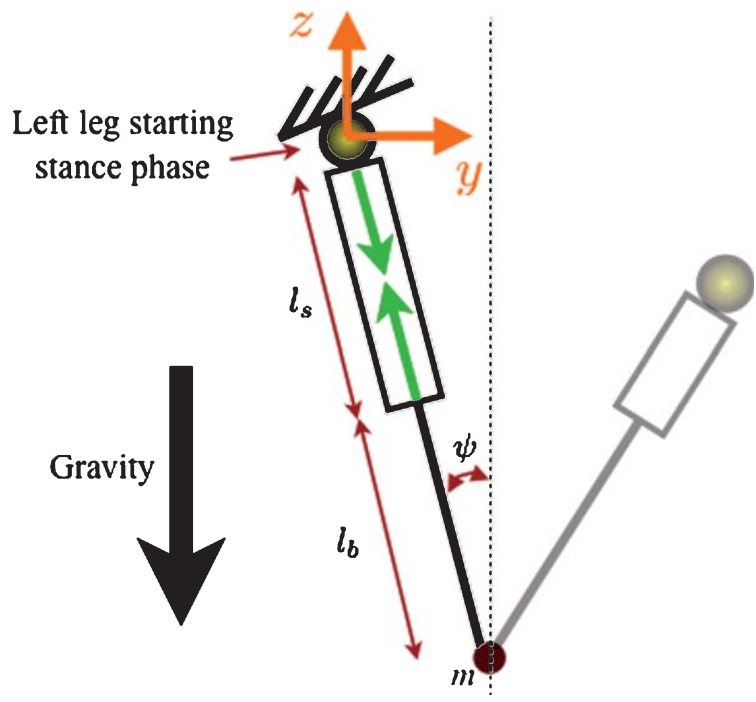

Fig. 2. The beginning of the world-switched climber's left leg stance phase. Sprawl angle is set, in this case, with respect to gravity. At the beginning of each side, irrespective of the former stance leg's angular position.

lateral forces, and the larger the sprawl angle, the larger the magnitude of those lateral forces.

The switching paradigm from the world-switched model corresponds to "smart" foot placement as opposed to the "blind" foot placement of the bodyswitched model. The world-switched climber requires more sensory feedback and physical dexterity than required for the body-switched climber. A climber behaving in accordance with the world-switched model, whether robot or animal, must have the sensory bandwidth to determine the vertical direction and the physical dexterity to position its legs with respect to that reference. Thus, this switching methodology seems to be more appropriate for larger animals that climb at a lower frequency (making stride-to-stride feedback more plausible) and are able to use more neural feedback (depending less on the "preflexes" that cockroaches seem to rely on [6], for instance). In the context of robotics, the distinction between these two types of models has been previously encountered in the analysis of sagittal plane runners [19] as representing a designer's choice between the "cheaper" sensorless, fixed sprawl angle behavior and a more "expensive" high-bandwidth, dexterous mechanism capable of implementing world-switched behavior. Understanding the advantages and deficits of the two schemes is of substantial importance in the design process.

\section{Actuator models}

Whereas the actuators in the Full-Goldman template specify limb length as a function of time (length changes apply a force to the body mediated by a wrist spring), our reduced order models employ actuators that exert forces as functions of state. This difference fundamentally alters the effect sprawl angle is able to have on the climber's vertical speed. While the Full-Goldman template's clock-driven actuation has no intrinsic power limitation and simply shortens the legs with a prescribed position trajectory, our force-based models have intrinsic power limitations and tradeoffs between force and velocity.

We now introduce the exact actuator functions which we will use to investigate our hypotheses. The first actuator model we construct corresponds to the motors used in DynoClimber [8, 14]. The second is a Blaberus discoidalis cockroach muscle model which has been used in simulations of a different biological template $[20,21]$. We must also specify a mechanism by which the motor's output torque causes a linear shortening (as occurs in our model). Similarly, a cockroach muscle does not pull on a point external from the cockroach's body; the muscle exerts force on an apodeme which relays that force to a segment of the exoskeleton, which, in turn, acts as a lever arm to apply force to the external world. Casting the muscle-skeleton interaction in engineering parlance, the exoskeleton acts as a transmission.

\subsection{Motor model and transmission}

We use the standard second order linear motor model (see, for instance, [22]). Under this model, a constant voltage applied over the motor terminals generates a linear relationship between rotational speed and torque. We describe torque as a function of rotational velocity for a motor at constant voltage,

$$
\tau_{\text {motor }}(f)=\tau_{\text {stall }} \cdot\left(1-\frac{f}{f_{\mathrm{nl}}}\right)
$$

in which $\tau_{\text {stall }}$ is the stall torque of the motor in $\mathrm{Nm}$, $f_{\mathrm{nl}}$ is the no-load speed of the motor in $\mathrm{Hz}$, and $f$ is the motor's rotational speed in $\mathrm{Hz}$.

This torque is converted to a linear force by means of a gear-reduction and a rack-and-pinion. This mechanism is chosen because its output force is a linear 
Table 1

Motor-powered model parameters

\begin{tabular}{llc}
\hline$m$ & point mass & $2.6 \mathrm{~kg}$ \\
$l_{b}$ & body length & $40 \mathrm{~cm}$ \\
$l_{s}$ & actuator stroke length & $13 \mathrm{~cm}$ \\
$f_{\mathrm{nl}}$ & no load rotational speed & $198.96 \mathrm{~Hz}$ \\
$\tau_{\text {stall }}$ & stall torque & $.321 \mathrm{Nm}$ \\
$G$ & gear ratio & $56.57: 1$ \\
$R$ & pinion radius & $4.14 \mathrm{~cm}$ \\
\hline
\end{tabular}

function of the input torque:

$$
F_{\text {motor }}\left(\tau_{\text {motor }}\right)=\frac{\tau_{\text {motor }} \cdot G}{R},
$$

where $G$ is the gear reduction used and $R$ is the radius of the pinion gear. The chosen parameters (see Table 1) represent the motors and gearing used in DynoClimber $[14,8]$.

Equipped with this actuator and a sprawl angle of roughly $10^{\circ}$, our model climbs in a qualitatively similar way to DynoClimber, the robot upon which these parameter values were based. The simulation forces and velocities agree with robot data in both phasing and magnitude. The simulated climber ascends roughly $24 \%$ more quickly than the robot. In ref. [23] we present a thorough comparison and attribute much of this speed discrepancy to actuator transmission choices; the simulated climber's rack-and-pinion enables faster climbing than the crank-slider mechanism employed on the robot (and simulated in ref. [23]).

\subsection{Muscle model overview and precedence}

Mathematical models for muscle function have been used since Hill's 1938 paper [24] derived-largely empirically - a force-velocity relationship for muscle contraction. In 1957, Huxley bolstered and refined Hill's equations while proposing a mechanistic view of muscle contraction: the Sliding-Filament Model [25]. The Sliding-Filament model additionally implies a relationship between force and muscle length which complements Hill's force-velocity relationship.

While more complex models exist that take into account (among other features) intramuscular geometry, the approach commonly taken when building a macro-scale simulation is to write muscle force $\left(F_{\text {mus }}\right)$ as

$$
F_{\text {mus }}=F_{1}(l) \cdot F_{\mathrm{v}}(v) \cdot F_{\mathrm{a}}(t) \cdot \alpha,
$$

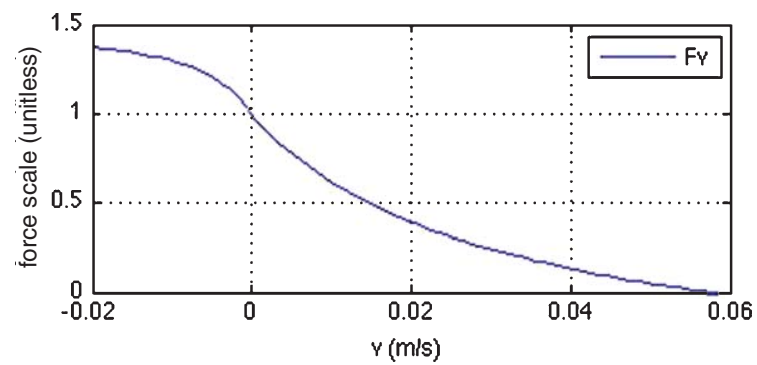

Fig. 3. A plot of the velocity force relationship, $F_{\mathrm{v}}$.

where $F_{\text {mus }}$ is the total muscle force, $F_{1}$ is the forcelength relationship, $F_{\mathrm{V}}$ is the force-velocity relationship, and $F_{\mathrm{a}}$ is the muscle's activation. We incorporate the scale factor $\alpha$ in order to represent multiple-leg recruitment. Similar models have been adopted to both analyze human gaits for prosthesis design [26], and to test prospective running templates [20].

\subsubsection{Force-velocity relationship}

The hyperbolic force-velocity relationship in a concentrically contracting muscle ${ }^{2}$ was described originally by Hill [24]. This force-velocity relationship is, for our purposes, the dominant factor in muscular force production. Using the model from Schmitt and Holmes [20], the muscle's force-velocity relationship is given by:

$$
F_{\mathrm{v}}(v)=\left\{\begin{array}{cc}
\frac{.52\left(v_{\max }-v\right)}{.52 v_{\max }+v}, & v \geq 0 \\
\frac{.114 v_{\max }-1.5 v}{.114 v_{\max }-v}, & v<0
\end{array}\right.
$$

Given the multiplicative form of $F_{\text {mus }}, F_{\mathrm{v}}$ is unitless and normalized such that its peak value is 1 . That is, $F_{\mathrm{v}}(0)=1$. The muscle model is scaled to the correct isometric contraction force through the force-length relationship $F_{1}$ and the scale factor $\alpha$. A plot of $F_{\mathrm{V}}$ is given in Fig. 3.

\subsubsection{Force-length relationship}

The essential feature of the force-length relationship is that near-peak force may be exhibited by the muscle at a range of lengths which lie roughly in the center of the muscle's total expansion range. We once again use

\footnotetext{
2 "Concentric" contraction refers to a muscle which is producing force and shortening simultaneously, "eccentric" contraction refers to force production during lengthening, and "isometric" contraction refers to force production by a muscle held at a constant length. We use the convention that a concentrically contracting muscle has $v>0$, while a lengthening muscle has $v<0$.
} 


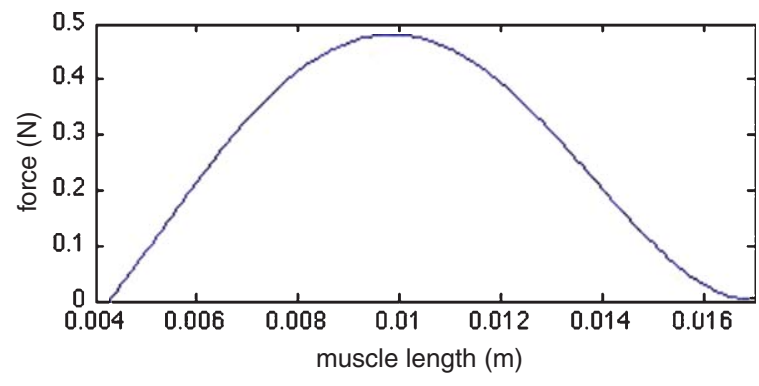

Fig. 4. A plot of the length force relationship, $F_{1}$.

the same function as [20],

$$
\begin{aligned}
F_{1}(l)= & F_{0} \cdot\left(4.435 \cdot\left(l / l_{0}\right)^{4}-16.46 \cdot\left(l / l_{0}\right)^{3}\right. \\
& \left.+18.28 \cdot\left(l / l_{0}\right)^{2}-5.333 \cdot\left(l / l_{0}\right)+0.1150\right),
\end{aligned}
$$

where $l_{0}$ is the muscle's "optimal" length, or the length at which the muscle is able to produce peak force. A plot of the length-force relationship is given in Fig. 4.

\subsubsection{Transmission}

Just as we did with our motor model, we ascribe a linear relationship between muscle force and an external force applied to the world. As described in [20], the actual cockroach muscle induces movement of the leg in an arc; we convert this arc to an effectively equivalent straight line via a linear approximation. We use similar numbers to [20] with muscle attached a length of $l_{\text {mus }}=.8 \mathrm{~mm}$ from the leg's joint for a leg length of $l_{\text {leg }}=8 \mathrm{~mm}$. The mechanical (dis)advantage from muscle to external world is, therefore 1:10. Looking at the force which the cockroach-modeled climber applies to the wall,

$$
F_{\text {ext }}(l, v)=\frac{\alpha}{10} \cdot F_{\mathrm{v}}(v) \cdot F_{1}(l) \cdot F_{\mathrm{a}}(t) .
$$

\subsubsection{Activation and scaling}

$F_{\mathrm{a}}$ is the muscle's activation - the extent to which the animal's nervous system is calling upon the muscle to contract. In [20], a template describing level ground running operates with a muscle model in which $F_{\mathrm{a}}$ is a smoothed "impulse" function in time. Running, however, is largely energetically conservative. In contrast, in our study of vertical climbing, the muscle must generate substantial power output as the climber's body gains gravitational potential energy constantly. Thus, we tune muscle activation to generate power output which results in animal-like climbing from our template.
In reality an animal's muscle functions in a work loop over the course of a periodic motion with submaximal activation for part of each stride, we make the approximation that each muscle is maximally activated $\left(F_{\mathrm{a}}=1.0\right)$ throughout its stance phase. A single actuator within our model represents the behavior of an entire tripod of cockroach legs; we therefore choose a compensating scale factor $\alpha$ which generates biologically accurate macro-scale behavior (forces, center of mass motion) from the climber. Simulating the worldswitched climber with a sprawl angle of $\psi=12^{\circ}$, we find that scale factor of $\alpha=1.3$ generates center-ofmass motions and vertical velocities whose magnitudes and phase relationships correspond roughly to those seen in actual cockroach data. See Fig. 5 for a comparison.

\section{Results}

We now examine simulation results for all four climbing model alternatives. We discuss body- and world- switched climbers, each paired with either a motor- or muscle- based actuator. For each switch and actuator pairing, we assess the two prospective consequences of sprawl: stability and actuator power output.

\subsection{Body-switched motor-powered climber}

In accord with the stability hypothesis, the bodyswitched climber demonstrates instability for sprawl angles below roughly $16^{\circ}$ (the threshold is strongly parameter dependent). Indeed, such a climber with a sprawl angle of $0^{\circ}$ goes unstable with only a slight lateral perturbation, as shown in the model's center of mass (COM) trajectory from the left plot in Fig. 6.

However, with $\psi$ above the apparent sprawl angle threshold, the climber converges to a singly-periodic (non-limping) steady-state gait. A climber with a large sprawl angle of $25^{\circ}$ is simulated, and its initial COM trajectory is given in in Fig. 6. Note that, despite an initial transient, the model eventually does converge to a stable gait; Fig. 6 shows the climber nearing, but not yet achieving, a period-1 gait.

\subsection{Body-switched muscle-powered climber}

The body-switched climber again demonstrates unstable climbing behavior with a sprawl angle of less than roughly $10^{\circ}$. Despite the substantial dif- 

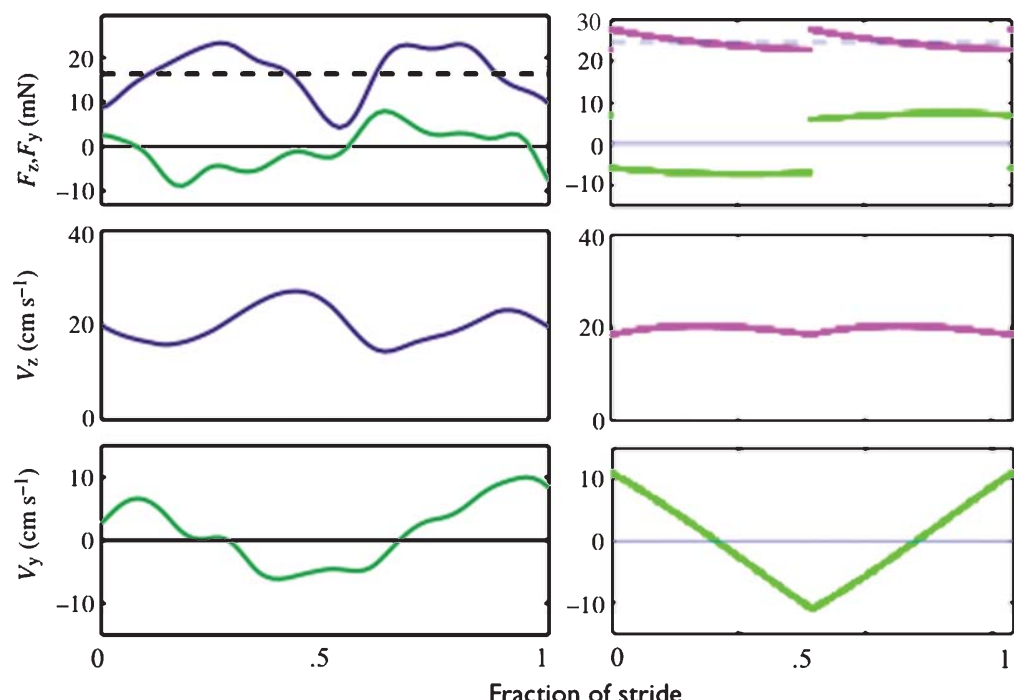

Fig. 5. Cockroach force and velocity on the left, world-switched template simulation on the right. Dotted lines represent body weight. Cockroach data taken from [2]. Note that while our template does not include a spring in series with the actuator, resulting in discontinuous force transitions, it climbs with similar velocity magnitude and phasing when compared to an actual cockroach.

ferences between muscle and motor dynamics, both body switched climbers demonstrate a dependence on sprawl angle to achieve stability.

Both the motor- and muscle-like actuators' power efficiencies are significantly degraded in the presence of the dynamical instabilities arising from insufficient sprawl in these body-switched simulations. Moreover, the substantial regions of instability depend upon not simply the sprawl angle, our central focus, but also upon $l_{b}$ (the climber's body-length), the model parameter with the least obvious relationship to a more anchored biological or robotic model. Preliminary numerical experience suggests this dependence arises in a complicated manner whose analysis lies well beyond the scope of the present paper. Thus, we are led to examine a variant switching model that relaxes the dynamical sensitivity of this reduced template at the "cost" of introducing into the model a more active sensory channel, again reminiscent of similar disjunctions in models of sagittal plane running [19].

Table 2

Muscle-powered model parameters

\begin{tabular}{llc}
\hline$m$ & point mass & $2.5 \mathrm{~g}$ \\
$l_{b}$ & body length & $4 \mathrm{~cm}$ \\
$l_{s}$ & actuator stroke length & $1.5 \mathrm{~cm}$ \\
$v_{\max }$ & maximum velocity of shortening & $0.0587 \mathrm{~m} / \mathrm{s}$ \\
$F_{0}$ & isometric contraction force & $0.46 \mathrm{~N}$ \\
\hline
\end{tabular}

\subsection{World-switched motor-powered climber}

The muscle-equipped world-switched climber, in contrast to its body-switched variant, converges to a stable, singly periodic gait over a large range of sprawl angles, from $0^{\circ}$ to over $45^{\circ}$. This range of stability makes the world-switched model attractive as a first-cut simulation vehicle for examining the power implications of body morphology and consequent lateral ("parasitic") forces, as sprawl can be varied over a large continuum of values without causing instability.

By simulating the climber over a range of sprawl angles and assessing its steady-state speed for each angle, we generate Fig. 7, a plot of vertical climbing speed versus sprawl angle. This data strongly confirms our hypothesis for the motor-powered climber: a nonzero sprawl angle can substantially improve climbing speed.

This result corroborates previous reports $[8,14]$ that increased sprawl angle improves climbing speed in simulation. In order to fully understand this phenomenon, we examine the range of actuator shortening velocities that are achieved at different sprawl angles, and how those changes in velocity affect power output. Figure 8 contains an unusual plot: the $x$-axis gives the actuator shortening velocity, while the $y$ axis plots the number of occurrences of a specific velocity for climbers with several different sprawl angles. That 

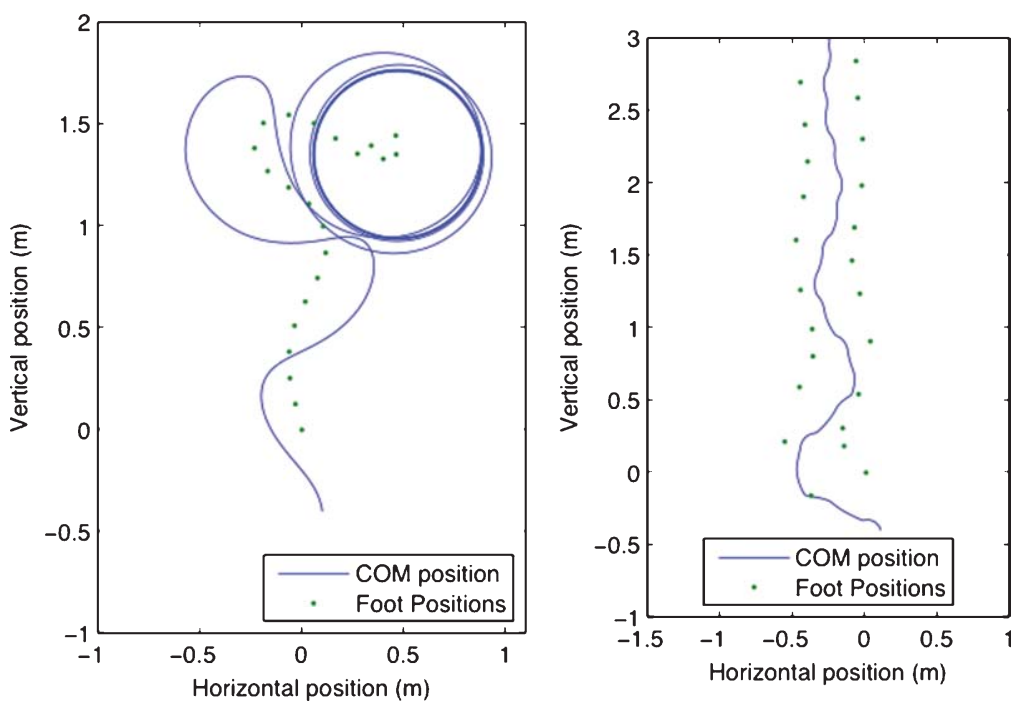

Fig. 6. The body-switched motor-powered climber's point mass traced along the $Y-Z$ plane as it climbs. Green dots show the attachment points used by the climber. The sprawl angle of the left climber is $\psi=0^{\circ}$ and for the right it is $\psi=25^{\circ}$. The climbers began with a slight lateral perturbation, yet evidently the non-sprawled climber becomes unstable, while the sprawled climber converges to a singly periodic gait.

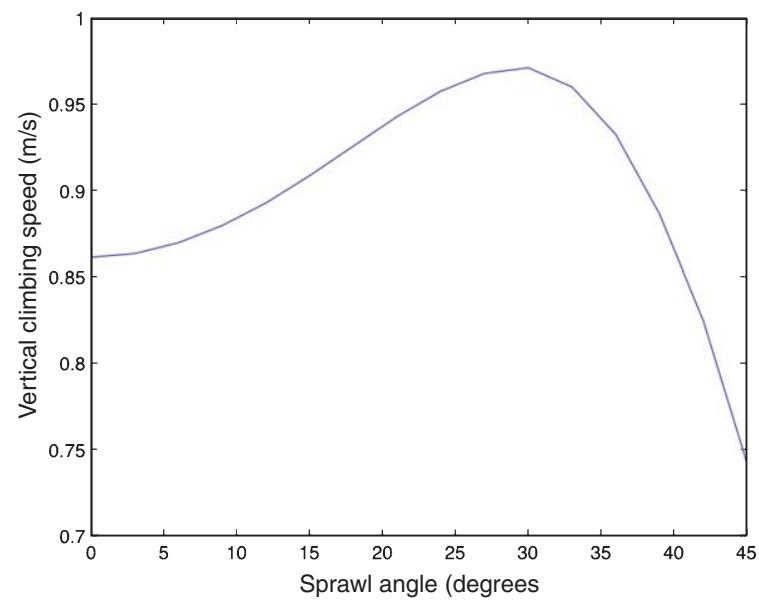

Fig. 7. Steady-state vertical climbing velocity plotted versus sprawl angle for the world-switched motor-powered climber. Note that a sprawl angle of roughly $30^{\circ}$ maximizes vertical climbing speed.

is, we examine histograms of shortening velocities. Superimposed is a scaled plot of actuator power output as a function of shortening velocity.

The effect of sprawl angle on shortening velocity is clear; the highest achieved shortening velocities in each stride monotonically increase as sprawl angle increases. Since a sprawled stride means that the actuator is fighting gravity at an angle instead of directly vertically, it is intuitive that the actuator should shorten more quickly, much as a car winding up a mountain road with switchbacks is able to travel at a greater speed than it could were it going directly up the hill.

Figure 7 indicates that a sprawl angle of approximately $30^{\circ}$ maximizes climbing speed, providing a roughly $12 \%$ improvement in vertical speed compared to a purely vertical climber. The cause of this phenomenon is not obvious from the histograms in Fig. 8. Indeed, the $30^{\circ}$ sprawled climber generates less power at its most frequently achieved speed than its lesssprawled relatives. Upon further inspection, though, a most important feature of the $30^{\circ}$ sprawled climber comes into view: its velocity profile is less concentrated, resulting in less time spent at lower velocities - and closer to the power peak. This phenomenon must be attributed to the interplay of the actuator and climber dynamics. At each stride transition, the climber is approaching its "old" point of attachment when the point of attachment instantaneously changes location. The climber's body continues to travel roughly toward the (former) location of the old attachment point, with the new attachment point an angular deviation away, resulting in an initially low shortening velocity.

The underlying actuator property which then results in more rapid climbing must not be overlooked: a motor must be geared to operate at a much higher speed than the speed at which it produces maximum power. For instance, the motor used in our synthetic actuator model (when driven at 30v), reaches its peak power 


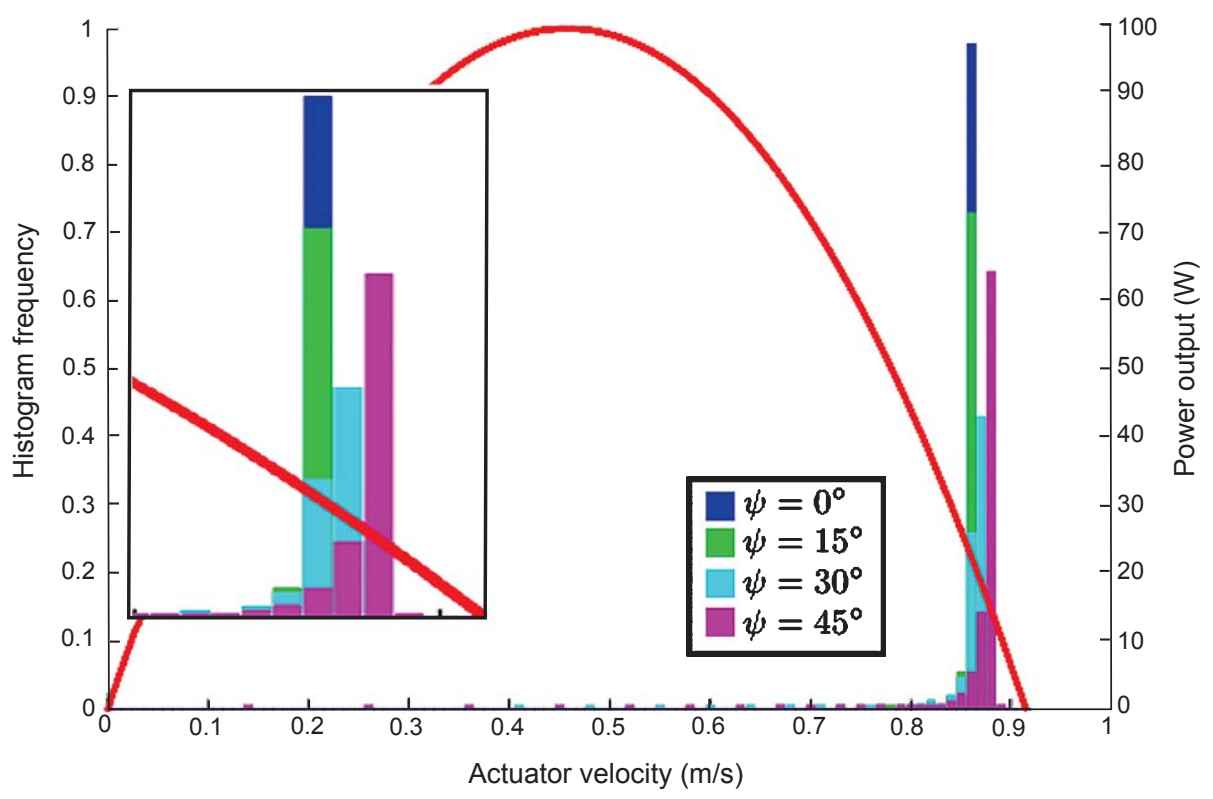

Fig. 8. The motor-powered climber's shortening velocities at four different sprawl angles are represented by separate histograms. Actuator power output is overlaid in red. The inset (note the modified aspect ratio) shows the histogram in more detail, making more evident the shifts caused by sprawl angle.

at a rotational speed of $99.5 \mathrm{~Hz}$, but destroys itself if it sustains a speed of less than $182 \mathrm{~Hz}$ when supplied with full voltage (see motor part number 118752 in [27]). This means that a climber driven by a motorprovided that the motor is geared such that it does not overload thermally - must operate on a sharply sloped region of the power curve, making the actuator's power output highly sensitive to variations in velocity. Decreased shortening velocity at the outset of each stride pushes the motor into a higher power regime and enables it to produce substantially more power.

\subsection{World-switched muscle-powered climber}

The muscle-based actuator used here does not alter the stability found in the world-switched motorpowered climber; once again, the climber demonstrates stability over a similar, large range of sprawl angles. We now analyze similar simulations with the cockroachlike actuated climber.

Once again, we examine a plot of vertical climbing speed versus sprawl angle (Fig. 9). In contrast to the synthetically actuated climber, the muscle-powered climber exhibits its fastest vertical climbing speed with a sprawl angle of $0^{\circ}$, and climbing speed monotonically decreases with sprawl angle beyond that. Interestingly,

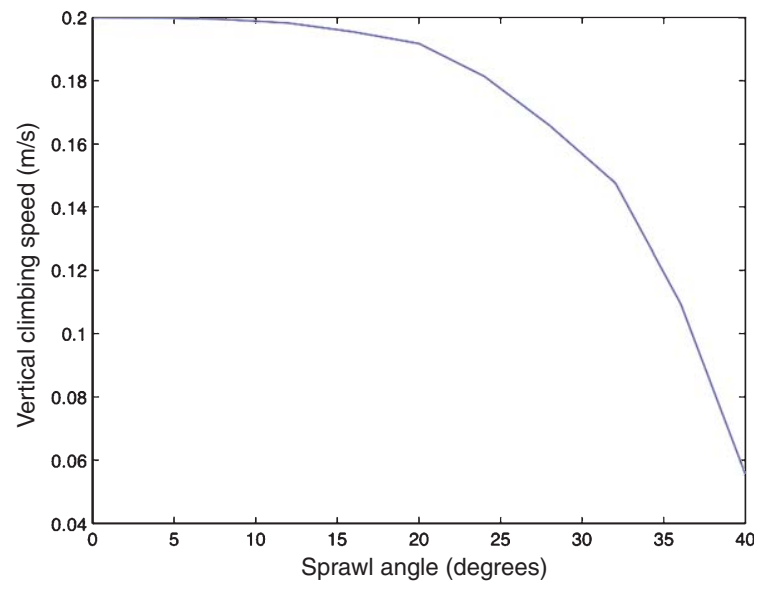

Fig. 9. Steady-state vertical climbing velocity plotted versus sprawl angle for the world-switched muscle-powered climber. Despite being tuned at a sprawl angle of $12^{\circ}$, a sprawl angle of $0^{\circ}$ maximizes vertical climbing speed.

up until a sprawl angle of roughly $15^{\circ}$, changes in sprawl angle do not have a dramatic effect on vertical climbing speed. Though the animal does not seem to benefit energetically from a sprawl angle, it also does not seem to pay a speed penalty if it employs (a biologically accurate) $10^{\circ}$ to $15^{\circ}$ sprawl. 


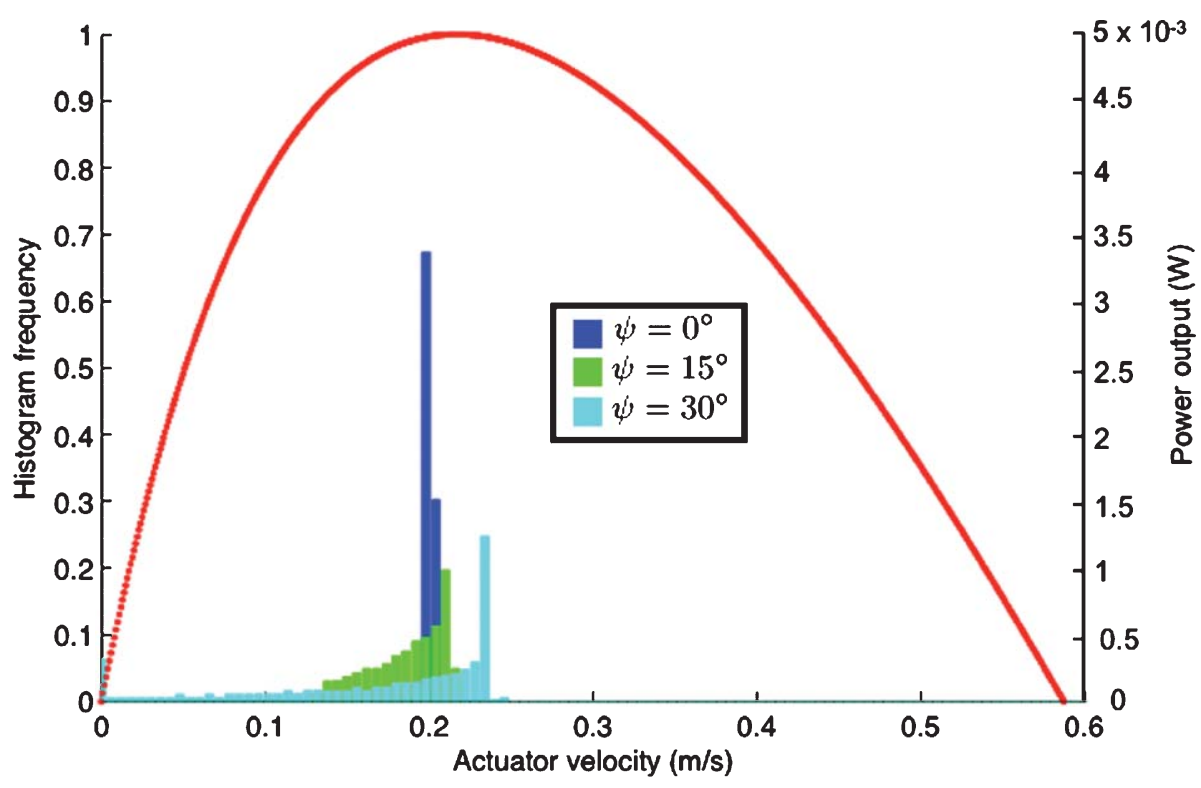

Fig. 10. The muscle-powered climber's shortening velocities at three different sprawl angles are represented by separate histograms. Actuator power output is overlaid in red.

Looking at the histogram and power versus shortening velocity plot in Fig. 10, the first note to make is the optimality of a cockroach's musculoskeletal design. Recall that only the cockroach muscle's activation was tuned to generate vertical velocities of roughly $20 \mathrm{~cm} / \mathrm{s}$, and all other parameters were selected to reflect physiological measurements of other researchers. It is remarkable, then, that for a level of activation which generates a biologically accurate climbing speed, the muscle is operating almost exactly at its peak power point. This finding is in line with existent data in muscle physiology. In [7], for instance, the frog musculoskeletal system is shown to be precisely tuned for a specific task: as a frog jumps, its muscles are operating at their maximum power output. Without evidence to the contrary, it is sensible to suppose that a cockroach in an evasive climbing situation would similarly achieve maximal power output. The fact that simulation indicates such a confluence partially validates both the muscle and transmission models used.

The next notable feature of Fig. 10 is that, just as in the motor-powered climber's case, increasing sprawl angle seems to increase the peak actuator speed as well as spread out the range of velocities achieved. However, unlike the motor-powered case, the musclepowered climber does not improve its total power output. This is attributable to two features of the cockroach-like climber's actuation: first, as mentioned before, the climber operates near the speed at which it generates peak power, even at a sprawl of $0^{\circ}$. The second facet of muscle is that its power curve is broader than that of a motor. Specifically, a muscle's forcevelocity curve is hyperbolic in shape, resulting in a flatter power peak than the motor with its linear torquevelocity profile and corresponding parabolic power curve. Perturbations from $0^{\circ}$, though they shift the operating regime of the actuator, are unable to push the muscle into a higher power regime and can function only to decrease total power output.

\section{Conclusion}

The results presented in this paper carry lessons both for robotic climbing, and for bio-inspired design in general.

First, with regard to stability, our results simulating the body-switched climber strongly corroborate even in this substantially simplified setting those of ref. [2], reflecting the critical role that sprawl angle plays in sensorless climbing stability. From these results, we conclude that for a robotic vertical climber to avoid using a sprawl angle, the robot must contain an adequate sensor suite as well as enough mechanical degrees of freedom to compute and actuate intelli- 
gent foot placement. On the other hand, a robot can rely on open-loop sensorless foot placement (with the mechanical and computational simplicity it entails) for stability as long as an adequately large sprawl angle is employed.

Second, our hypothesis - that sprawl angle can positively affect actuator power output-is confirmed in the robotic case, but rejected in muscle-powered simulation. Our robotic climber stands to benefit substantially in vertical speed if the correct sprawl angle is chosen. The direct application of this result is, of course, that a robotic climber's sprawl angle may be employed to substantially increase vertical climbing speed, while possibly affecting dynamic stability.

We and our collaborators have derived significant engineering benefit from bioinspired robot design as well as substantial biological insight from studying physical (robot) models of locomotion [3]. This study, however, offers a useful cautionary tale for both styles of investigation. These simulations suggest that while a cockroach's power production is not penalized for sprawled climbing, the sprawl likely does not improve climbing speed. Thus, the benefit of its pendulous swaying seems to likelier to emerge as a consequence of the sensory deprived high-stride frequency climbing regime ${ }^{3}$. Conversely, given that vestibular sensors of a kind required to implement world-frame feedback in the relevant highstride frequency regime are now far more readily available commercially and algorithmically effective [28] than a decade ago when RHex [9] first appeared, it may be the case that sprawled posture robotic climbing must derive its primary justification from power rather than dynamical considerations. Both perspectives lend further weight to the arguments against blind copying of nature by engineers and of uncritical appeal to physical models by biologists.

\section{Acknowledgments}

This paper was presented in a similar form and with the same name at the 2010 International Conference on Applied Bionics and Biomechanics. We thank the reviewers from that conference for their insightful

\footnotetext{
${ }^{3}$ In pursuing this hypothesis, it will be crucial to examine the sensory capabilities of lizards which also exhibit pronounced lateral motions while climbing [2].
}

comments. This work was supported in part by the US National Science Foundation under FIBR grant 0425878 and by the US National Institute of Health under grants AR38404 and AR46125.

\section{Appendix}

The dynamics of both world- and body- switched models are identical between the beginning and end of a stride, only differing during transition. In cartesian coordinates, with the climber's foot placement at the origin, the climber's mass obeys the following equations of motion:

$$
\left[\begin{array}{l}
\ddot{x} \\
\ddot{y}
\end{array}\right]=\ddot{p}=\left[\begin{array}{c}
\frac{-x F_{\mathrm{a}}(p, \dot{p})}{m \sqrt{x^{2}+y^{2}}} \\
\frac{-y F_{\mathrm{a}}(p, \dot{p})}{m \sqrt{x^{2}+y^{2}}}-g
\end{array}\right]
$$

This equation specifies the climber's foot placement within a given stride (i.e. $l_{b} \leq \sqrt{x^{2}+y^{2}} \leq l_{s}+l_{b}$ ); one stride transitions to the next when $\sqrt{x^{2}+y^{2}} \leq l_{b}$.

The two models differ in their treatment of stride transitions. To describe this behavior, we use a subscript $f$ to denote a variable's final state at the end of a stride, with a subscript $i$ denoting its initial state at the beginning of the subsequent stride.

The world-switched hybrid transition, then, is given by

$$
\left[\begin{array}{c}
x_{i} \\
\dot{x}_{i} \\
y_{i} \\
\dot{y}_{i}
\end{array}\right]=\left[\begin{array}{c}
(-1)^{n}\left(l_{b}+l_{s}\right) \sin (\psi) \\
\dot{x}_{f} \\
-\left(l_{b}+l_{s}\right) \cos (\psi) \\
\dot{y}_{f}
\end{array}\right],
$$

where $n$ counts strides to keep track of left and right steps.

To specify the body-switched transition, we introduce an angular coordinate,

$$
\theta=\arctan \left(\frac{x}{-y}\right)
$$

and use it to write down the body-switched transition:

$$
\left[\begin{array}{c}
x_{i} \\
\dot{x}_{i} \\
y_{i} \\
\dot{y}_{i}
\end{array}\right]=\left[\begin{array}{c}
\left(l_{b}+l_{s}\right) \sin \left(2(-1)^{n} \psi+\theta_{f}\right) \\
\dot{x}_{f} \\
-\left(l_{b}+l_{s}\right) \cos \left(2(-1)^{n} \psi+\theta_{f}\right) \\
\dot{y}_{f}
\end{array}\right]
$$




\section{References}

[1] R.J. Full and D.E. Koditschek, Templates and anchors: Neuromechanical hypotheses of legged locomotion on land, Journal of Experimental Biology 202(23) (1999), 3325-3332.

[2] D.I. Goldman, T.S. Chen, D.M. Dudek and R.J. Full, Dynamics of rapid vertical climbing in a cockroach reveals a template, Journal of Experimental Biology 209 (2006), 2990-3000.

[3] P. Holmes, R.J. Full, D. Koditschek and J. Guckenheimer, The dynamics of legged locomotion: Models, analyses, and challenges, SIAM Review 48(2) (2006), 207-304.

[4] R. Blickhan and R.J. Full, Similarity in multilegged locomotion: Bouncing like a monopod, Journal of Comparative Physiology 173(5) (1993), 509-517.

[5] J. Schmitt and P. Holmes, Mechanical models for insect locomotion: Dynamics and stability in the horizontal plane i. theory, Biological Cybernetics 83(6) (2000), 501-515.

[6] T. Kubow and R. Full, The role of the mechanical system in control: A hypothesis of self-stabilization in hexapedal runners, Philosophical Transactions of the Royal Society of London Series B-Biological Sciences 354(1385) (1999), 849-861.

[7] G. Lutz and L. Rome, Built for jumping: the design of the frog muscular system, Science 263 (1994), 370-372

[8] J. Clark, D. Goldman, P. Lin, G. Lynch, T. Chen, H. Komsuoglu, R. Full and D. Koditschek, Design of a bioinspired dynamical vertical climbing robot, in Proceedings of Robotics: Science and Systems, Atlanta, GA, USA, 2007.

[9] R. Altendorfer, N. Moore, H. Komsuoglu, M. Buehler, J. Brown, H.B. Brown, D. McMordie, U. Saranli, R. Full and D.E. Koditschek, Rhex: A biologically inspired hexapod runner, Autonomous Robots 11(3) (2001), 207-213.

[10] D.E. Koditschek, R.J. Full and M. Buehler, Mechanical aspects of legged locomotion control, Arthropod Strct Dev 33 (2004), 251-272.

[11] R. Altendorfer, U. Saranli, H. Komsuoglu, D. Koditschek, H. Brown, M. Buehler, N. Moore, D. McMordie and R. Full, Evidence for spring loaded inverted pendulum running in a hexapod robot, in Experimental Robotics VII, vol. 271, Spring-Verlag, Berlin, 2001, pp. 291-302.

[12] S. Jensen-Segal, S. Virost and W. Provancher, Rocr: Dynamic vertical wall climbing with a pendular two-link mass-shifting robot, in Proceedings of IEEE ICRA, Pasadena, CA, 2008.

[13] A. Degani, A. Shapiro, H. Choset and Mason, A dynamic single actuator vertical climbng robot, in Proceedings of IEEE IROS, 2007, pp. 2901-2906.

[14] J.E. Clark, D.I. Goldman, T.S. Chen, R.J. Full and D. Koditschek, Toward a dynamic vertical climbing robot, in
International Conference on Climbing and Walking Robots (CLAWAR), vol. 9, Professional Engineering Publishing Brussels, Belgium, 2006.

[15] J.E. Clark and D.E. Koditschek, A spring assisted one degree of freedom climbing model, Lecture Notes on Control and Information Sciences, 2006, pp. 43-64.

[16] W.J. Schwind and E.D. Koditschek, Characterization of monoped equilibrium gaits, in Proceedings of the 1997 IEEE International Conference on Robotics and Automation, Albuquerque, New Mexico, 1997.

[17] D. Koditschek and M. Buehler, Analysis of a simplified hopping robot, International Jouranl of Robotics Research $\mathbf{1 0}(6)$ (1991), 587-605

[18] G.A. Lynch, J.E. Clark and D.E. Koditschek, A self-exciting controller for high-speed vertical running, in Proceedings of IEEE IROS St. Louis, 2009

[19] R. Altendorfer, D.E. Koditschek and P. Holmes, Stability analysis of legged locomotion models by symmetry-factored return mpas, International Jouranl of Robotics Research 23(11) (2004), 979-1000.

[20] J. Schmitt and P. Holmes, Mechanical models for insect locomotion: active muscles and energy losses, Biological Cybernetics 89 (2003), 43-55.

[21] R. Kukillaya and P. Holmes, A model for insect locomotion in the horizontal plane: Feedforward activation of fast muscles, stability, and robustness, Journal of theoretical biology 261(2) (2009), 210-226.

[22] I. Poulakakis, J.A. Smith and M. Buehler, Experimentally validated bounding models for the Scout II quadrupedal robot, in ICRA, 2004, pp. 2595-2600.

[23] G.A. Lynch, L. Rome and E.D. Koditschek, Sprawl angle in sim-plied models of vertical climbing: implications for robots and roaches, in Proceedings of the 2010 International Conference on Applied Bionics and Biomechanics, 2011.

[24] A. Hill, The heat of shortening and the dynamic constants of muscle, Proc R Soc Lond B, no. 126, 1938, pp. 136-195.

[25] A. Huxley, Muscle structure and theories of contraction, Prog Biophysics and Biophysical Chemistry 7 (1957), 255-318.

[26] K. Endo and H. Herr, Human walking model predicts joint mechanics, electromyography and mechanical economy, in Proceedings of IEEE IROS St. Louis, 2009.

[27] A.G. Maxon Motor, Briinigstrasse 220 P.O.Box 2636072 , Sachseln Schweiz, Maxon Motor Catalog, 2010.

[28] P.C. Lin, H. Komsuoglu and D.E. Koditschek, Sensor data fusion for body state estimation in a hexapod robot with dynamical gaits, IEEE Transactions on Robotics (TRO) 22(5) (2006), 293-943. 

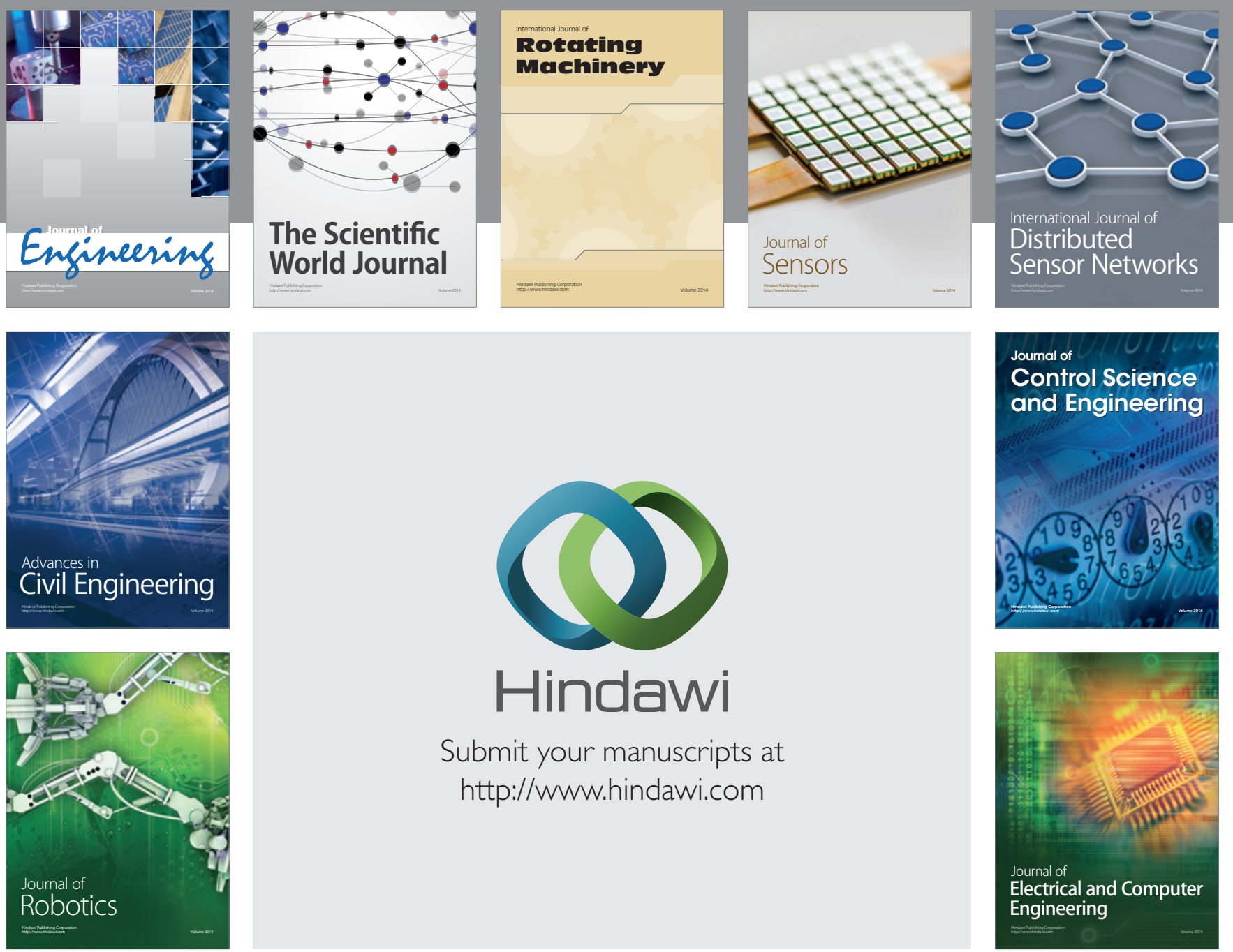

Submit your manuscripts at

http://www.hindawi.com
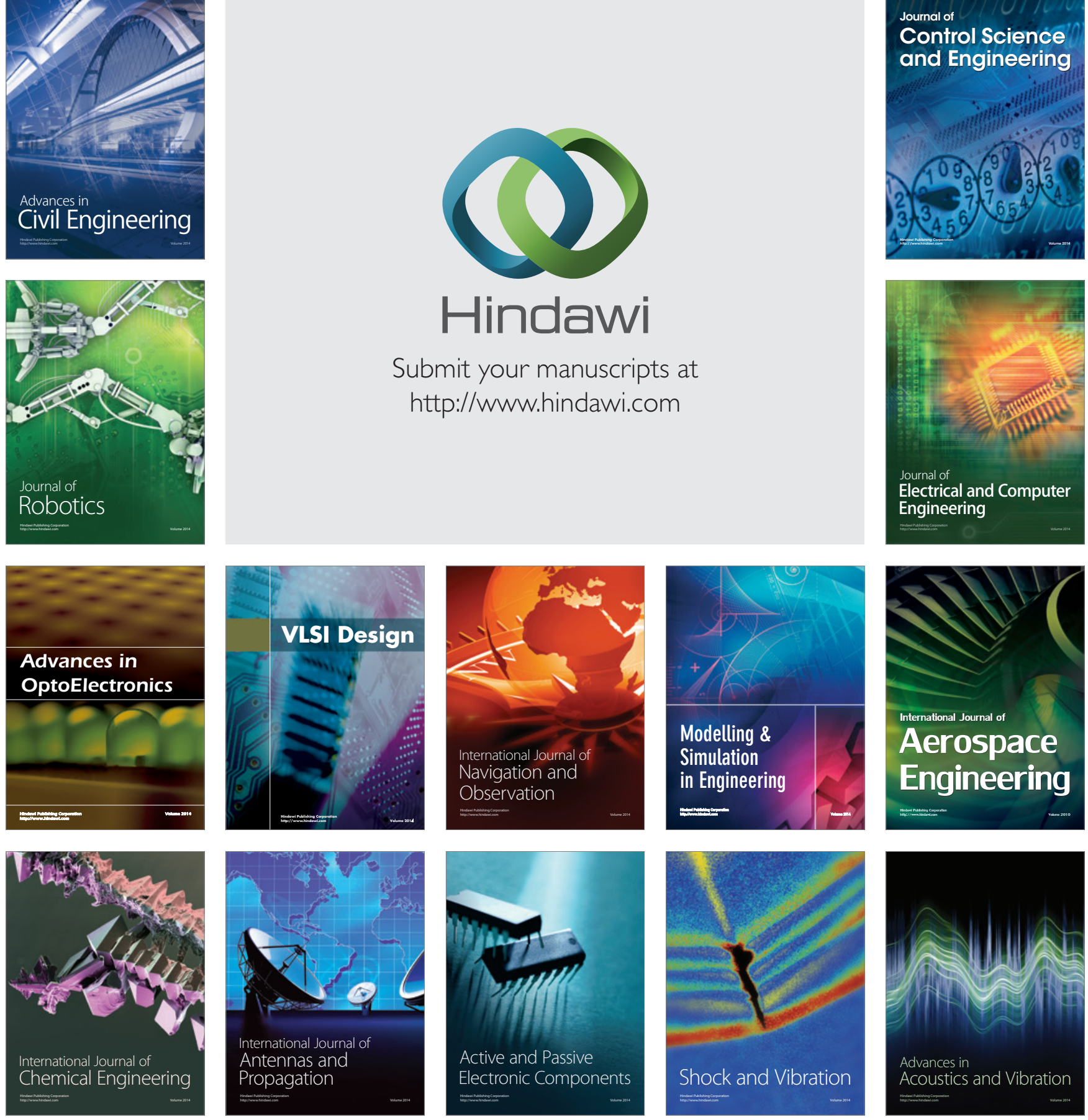